\title{
Development of Prototype Simplified Neutron Scatter Camera for Nuclear Safeguards Applications
}

\author{
Taylor Harvey $^{1}$ and Andreas Enqvist ${ }^{1}$ \\ ${ }^{1}$ University of Florida, Gainesville, Florida, United States of America \\ Corresponding author: taylor.harvey250@ufl.edu
}

\begin{abstract}
Neutron scatter cameras are a type of directional neutron detectors that rely on multiple neutron scatters to generate images that can reveal the direction and distribution of neutron sources. Fast neutron cameras which have recently been developed rely on multiple detector volumes and make use of neutron time-of-flight measurements. These designs, though effective in localizing the source direction, relies on a large amount of detection and electrical equipment, thus increasing size, cost, and complexity of the systems to unreasonable levels for some applications. This project seeks to develop a compact scatter camera that is less expensive than systems relying on multiple detector volumes. Crucially, two components and capabilities are needed to achieve this: fast scintillation detection materials and picosecond electrical pulse timing. Utilizing such electronics, distinguishing between scintillation light pulses generated by the same neutron within one detector volume is possible. An MCNPX-PoliMi model of such a system has been developed to guide prototype designs. A cube of EJ-230 fast plastic scintillator and six photomultiplier tubes (PMTs) were used to construct the prototype camera that localizes neutron sources based on the principle of cone back projection. Prototypes of the system in one, two, and three dimensions have shown promising initial results when coupled with a script that algorithmically identifies candidate neutron double scatter events and back projects probability cones in the direction of possible sources. Imaging resolution/quality, double scatter efficiency, and cost for the system are quantified. Paths forward for further improvement of a future system based on this camera' operating principles are discussed.
\end{abstract}

Keywords - neutron imaging, neutron detection, international safeguards

\section{INTRODUCTION}

The field of nuclear safeguards seeks to stop the spread of nuclear weapons by developing institutional, legal, and technical mechanisms intended to prevent the misuse of nuclear materials and technology $[1,2]$. Essential to the nuclear safeguards paradigm is the use of nuclear material measurement technologies to monitor and verify the quantity, identity, and movement of radioactive sources relevant to both peaceful and weapons programs. Of particular interest is Special Nuclear Material (SNM), such as uranium and plutonium, which can be monitored and identified using a variety of neutron detection techniques [3]. Much of the focus of nuclear material measurement technologies for safeguards is on destructive analysis (DA) and nondestructive assays
(NDA) of nuclear material. Both DA and NDA techniques seek to determine properties of nuclear material through chemical, spectroscopic, or other means to make quantities known for material and accounting purposes. This suite of techniques can reveal, often in fine detail, the "what" and "how much" of a material of interest but cannot give information about the location or distribution of a source if these facts are unknown. A new class of radiation detectors, known as directional or imaging detectors, seek to further the international safeguards technical repertoire by providing spatial information on radioactive sources.

Neutron scatter cameras (NSCs) operate by detecting two consecutive elastic scatters of a single fast neutron emitted by a nearby source. The kinematic principle involved in determining the original particle trajectory from two consecutive scattering events is similar to the operating principle of Compton cameras, though NSCs use fast neutrons rather than gamma rays [4]. The detector active volumes present in all currently existing NSCs use either organic liquid or plastic scintillators. The initial trajectory of individual neutrons is determined by finding the approximate $(\mathrm{x}, \mathrm{y}, \mathrm{z})$ position of both the first and second scatter within the scintillator volume. The position of each scatter is found by either using spatially separated volumes of scintillators, or by comparing the relative position and timing of the arrival of light to a series of photodetectors coupled to the same scintillator volume. Spatially separated arrays of scintillators may be arranged in either multiple planes or in a radiallysymmetric arrangement. Mascarenhas et al. [5], Goldsmith et al. [6], and Weinfurther et al. [7] provide detailed technical discussions of the kinematics of scattering in plane-based, radial, and single volume designs respectively. After the determination of two consecutive scatter positions and the determination of the energy deposited in the first scatter, probability cones encompassing the possible trajectories of individual neurons can be back projected in 3-D space. The region of space where the surfaces of the all the back projected cones overlap is interpreted to be the most likely direction of the neutron source.

Advances in both fast light pulse plastic scintillator materials and sub-nanosecond digitizer have allowed for the construction of compact or single volume scatter cameras with high efficiencies and accurate angular imaging resolutions. Currently developed systems rely on photoelectronics and/or segmented designs that lead to systems with high unit costs and high amount of data throughput from many digitized channels. The "simplified" prototype neutron scatter camera discussed in this paper proposes a design that is mobile, simple to use, and affordable while still providing useful data 
regarding the location, distribution and identity of neutronemitting sources.

\section{EXPERIMENTAL DESIGN}

\section{A. Detector Construction and Calibration}

An MCNPX-PoliMi model of a cube-shaped plastic scintillator volume was developed to guide the parameters of the simplified neutron scatter camera system. Simulation guided the selection of a 6" side length cube of Eljen's EJ-230 fast plastic scintillator for the detection medium, six Electron Tube $9821 \mathrm{~KB}$ photomultiplier tubes for the photodetectors, PSI's DRS4 digitizer for pulse digitization, and a custom MATLAB script for the data processing. These materials were selected to allow for the resolution of consecutive same-neutron scatters that appear only nanoseconds apart in time.

To create the back-projected probability cones needed to image neutron sources, the camera must be able to determine the scatter positions of neutron scatters one and two, the energy deposited in the first neutron scatter, and the time of flight between scatters one and two. In the simplified design, determination of the neutron scatter positions is done by comparing the amount of light arriving at photomultiplier tubes coupled to opposing scintillator cube faces for voltage pulses algorithmically tagged as neutron double scatters. The double scatter pulse heights are proportional to the amount of light arriving at each PMT, which is itself proportional to the sighting factor from the scatter position to PMT photocathode, as described by the equation:

$$
P_{n}=L_{n} \log \left(N P_{n 1} / N P_{n 2}\right)
$$

Where $P_{n}$ is the position along the $n$-dimension of the cube scintillator, $L_{n}$ is the length of the scintillator in the $n$ dimension, and NP is the number of photons arriving at the two photodetectors oriented normal to the n-dimension. The scintillation position is localized in all three coordinates when this light intensity ratio comparison is done simultaneously across all six photodetectors. The energy deposited in the first neutron scatter is found by summing the light arriving at all six PMTs, determining the electron equivalent energy deposition corresponding to that amount of light, and calculating the proton recoil energy by using the electron equivalent value with the characteristic light curve for the scintillator material. The time of flight between the two neutron scatters is found by averaging the time between "peaks" corresponding to primary and secondary same neutron scatters in the pulse streams produced by each of the six PMTs. The relevant kinematic equation for cone back-projection are:

$$
\begin{aligned}
E_{s} & =\frac{1}{2} m\left(\frac{d}{t}\right)^{2} \\
E_{i} & =E_{f}+E_{s} \\
\theta_{n} & =\sin ^{-1} \sqrt{\frac{E_{f}}{E_{i}}}
\end{aligned}
$$

Where $E_{f}$ is the energy deposited by the first neutron scatter, $E_{s}$ is the neutron energy after the first scatter, $E_{i}$ is the kinetic energy of a neutron from the source, $\mathrm{m}$ is the mass of the neutron, $\mathrm{t}$ is the time between scatters 1 and $2, \mathrm{~d}$ is the distance between scatter positions 1 and 2 , and $\theta_{\mathrm{n}}$ is the central angle of the back projected cone of possible source locations. Figure 1 summarizes the kinematics of double neutron scattering in the simplified design, and Figure 2 shows a photograph of the prototype simplified neutron scatter camera system.

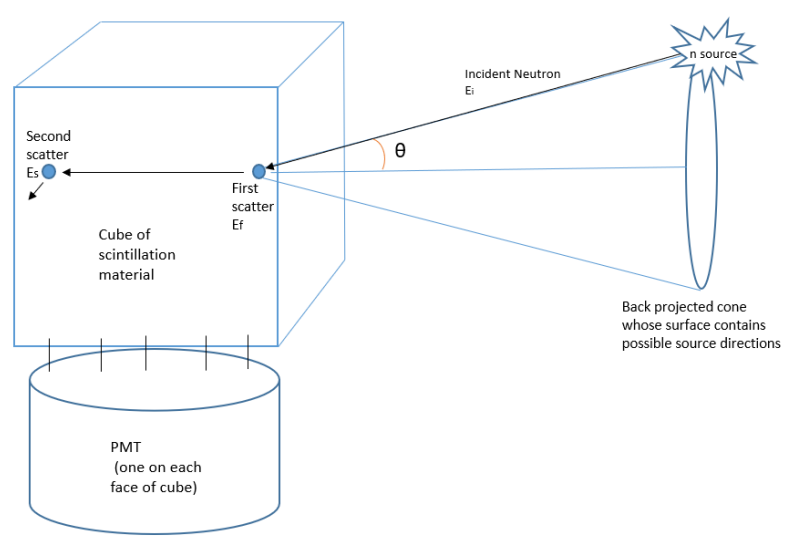

Fig. 1. Basic operating principle of a monolithic neutron scatter camera. Neutrons emitted by nearby sources must scatter at least twice in the scintillator volume. The scattering positions are determined by comparing the amount of light arriving at each of the six PMTs mounted to the six surfaces of the scintillator. By solving a series of kinematic equations, a probability cone whose surface contains the possible trajectories of the neutron can be backprojected into 3-D space. Many correctly back-projected cones will converge to the source location.

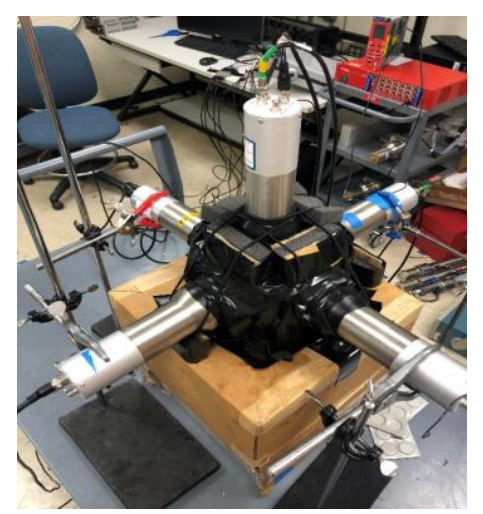

Fig. 2. Three-dimensional neutron scatter camera prototype. The PMT coupled to the bottom face is not visible as it is resting inside of the cardboard box support. Photo courtesy of the authors.

The camera was gain calibrated for neutron scatter position by finding maximum and minimum light ratios through simulation and assuming a log-linear relationship across the detector dimensions. Energy calibration was performed by plotting the 90 percent height Compton edge values for ${ }^{60} \mathrm{Co}$, ${ }^{137} \mathrm{Cs}$, and ${ }^{22} \mathrm{Na}$ gamma spectra measured with each of the PMTs used with the camera. Proton recoils for each neutron scatter event were found by performing neutron time-of-flight measurements using the EJ-230 material and comparing them with published results with the same material [8]. The system was time calibrated by comparing the pulse rise time response between two of the system PMTs, resulting in a system timing uncertainty of $\sigma_{\mathrm{t}}=0.82 \mathrm{~ns}$. Figures 3,4 , and 5 display the relevant calibration curves used in finding the kinematic data 
from each neutron double scatter event.

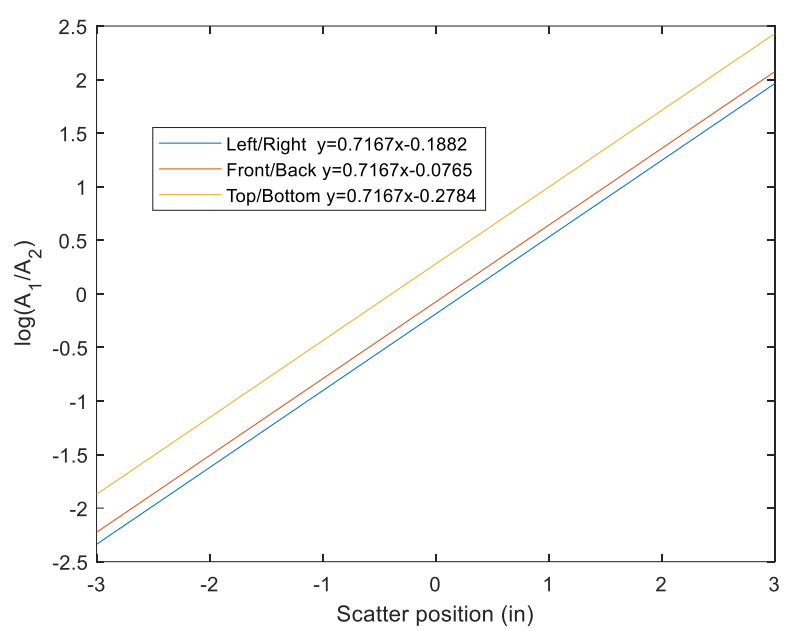

Fig. 3. Position calibration curves for opposite PMT neutron scatter events for the left/right (x), front/back (y), and top/bottom (z) PMT pairs. The linear curves were fit to the maximum/minimum achievable light ratios from the geometric end points at -3 and 3 inches determined from simulation.

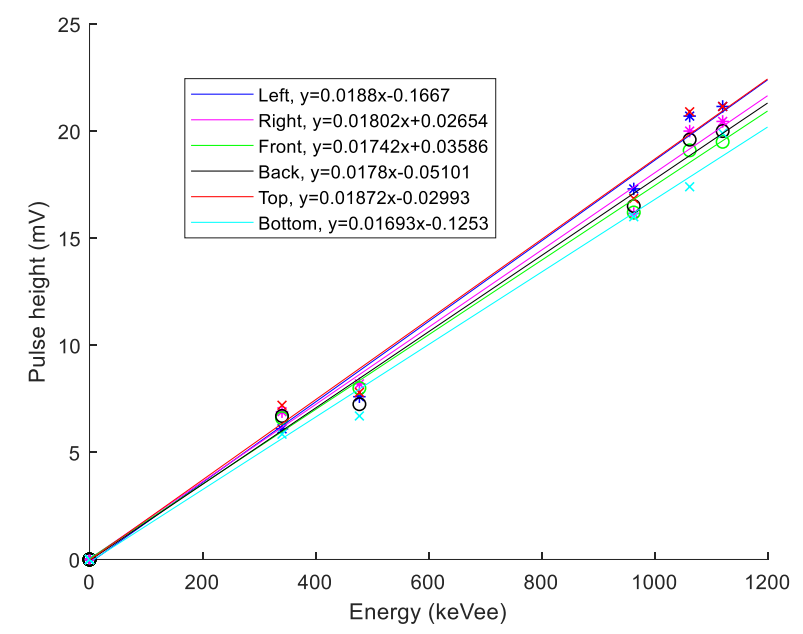

Fig. 4. Linear energy calibration curves for each of the six PMT used in the scatter camera. The five $90 \%$ height Compton edge values for each PMT are plotted along with lines of best fit forced to pass through zero.

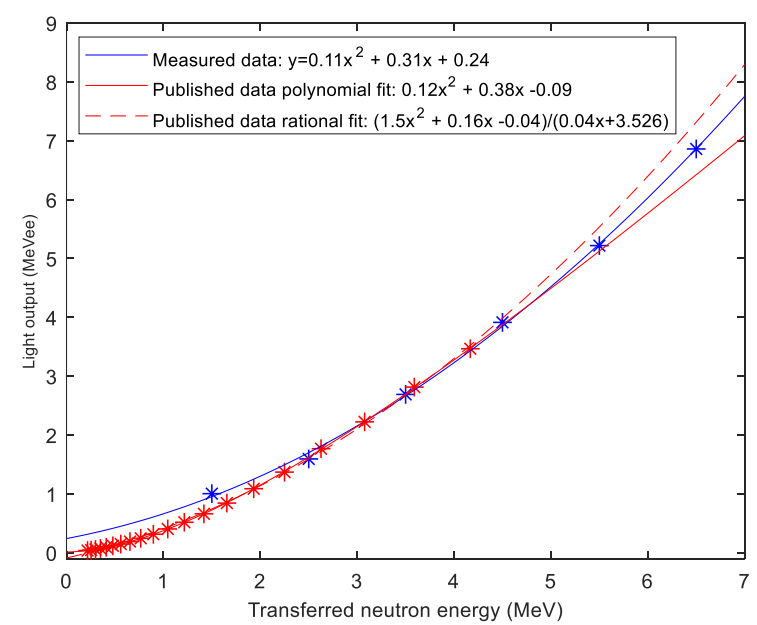

Fig. 5. Light functions for EJ-230 material from measured data and previously published data [8]. Second-degree polynomial and two-over-one rational fits were used to generate the light functions. Deviation between the two data sets can be attributed to different detector shapes and different methods of PHD edge finding.

\section{B. Double Scatter Identification Algorithm}

The overall goal of the algorithm used in tandem with the simplified NSC is to read in voltage pulse arrays produced by the system, determine parameters of the pulses based on their shape, height, timing, and relative prominence, use these parameters to estimate the positions and energy depositions associated with neutron double scatter events, and finally use these scattering parameters to generate neutron images based on the principle of back-projection. The data files needed to complete this sequence are necessarily large, with a sixchannel pulses set with one million pulses at a sampling rate of 5 GSPS per channel resulting in a data file size of approximately $12 \mathrm{~GB}$. To prevent memory allocation issues when running the script in MATLAB, the script was broken into three main sections which can be run sequentially and independently. The first section reads the data files output by the PSI DRS4 digitizer into pulse arrays that can be manipulated by MATLAB. The second section takes those arrays and extracts energy, timing, and shape data from them to identify pulses that may have resulted from double scatter events. The third section uses the double scatter candidate pulses to form a neutron image through cone back-projection and improves the image through a process of cone downselection based on an initial source direction guess vector. The overall script runs most efficiently and experiences the least memory allocation related slowdown when the first script section is used to read in a small fraction of the total pulses from the data file and transfers the read pulses to the second section for selection of double scatter candidates. Once the second section finds the double scatter candidates for the sample of the total pulses, the non-candidate pulses can be deleted, and the first section can be run again to import the next sample of pulses. This loop between sections I and II can continue until the original data file has been completely read. Next, with an array of double pulse candidates found, the imaging section can be run once to generate a single neutron image or be run iteratively to generate a series of images with different down-selection parameters, such as energy disposition cutoff, choice of proton recoil light curve, second scatter criterion, second pulse timing window length, and size of guess vector down-selection region. A flowchart demonstration the overall data flow in the script is shown in Figure 6. 


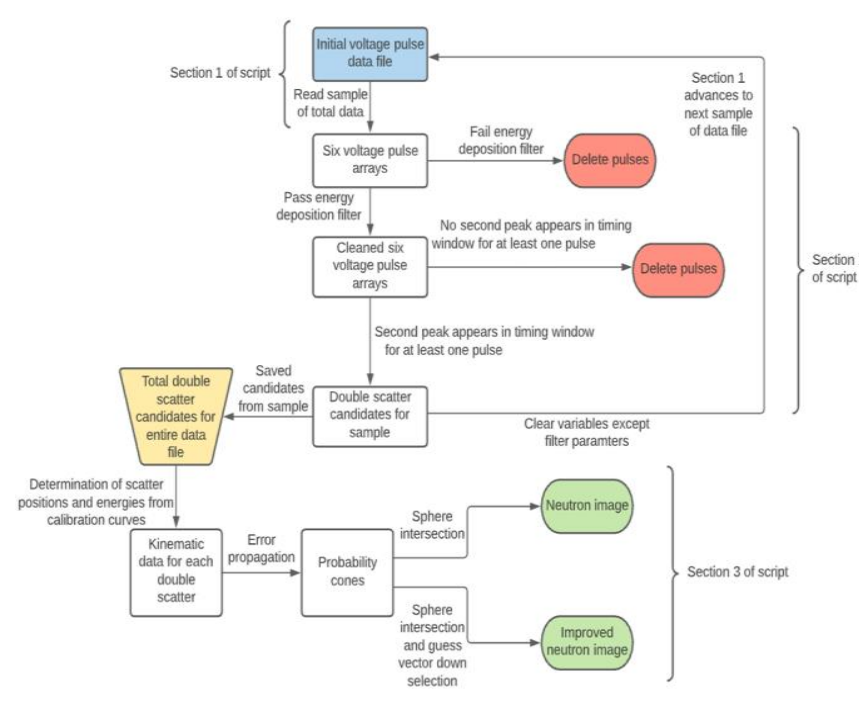

Fig. 6. Flowchart of data in the MATLAB script used in the algorithm that reads and processes neutron imaging data from the prototype simplified NSC.

The second section of the script is the most computationally taxing, mostly due to the 'findpeaks' function used in identification of pulse features that could correspond to second neutron scatters. This section of the script opens with the definition of a set of user-set parameters for the sensitivity of the second scatter feature search. These include the selection of which data set and fit to use for the light curve, the peak prominence ratio cutoff for the two most prominent peaks in a pulse, the first scatter energy deposition cutoff, a pure pulse height cutoff value to remove noise, and second peak window start and ending time values. After defining these parameters, the raw pulses are inverted, adjusted for gain pedestal to move each pulse to a baseline of zero, and cut down from 1024 data points to 351 data points each. These steps are taken to reduce the amount of data processing necessary for each pulse and to ensure the built-in MATLAB functions work properly. The maximum value for each pulse is then found and saved in six separate arrays. These maximums are then used to find log pulse height ratios for the left/right, front/back, and top/bottom PMT pairs. The pulse height values are then converted from digitizer units to millivolts and the maximum peak values are applied to the energy calibration curves presented in Figure 4 to yield first scatter deposition values in MeVee for each PMT. The MeVee value corresponding to each of the six PMTs for a single event is then averaged to yield the "true" electron-equivalent energy deposition value for the scattering events. Averaging across the PMTs removes any scatter position-related bias related to asymmetric light arrival at the various photocathodes. The electron-equivalent energy values are then applied to the inverse of one of the light curve fits presented in Figure 5. This yields an array of first scatter neutron energies. In actuality, some portion of these pulses are caused by gamma scatters or cosmic rays, though those events should be removed by the later double scatter and source direction guess vector filters. The script then removes all pulses correlated with neutron deposition energies of less than the cutoff value. Altering this cutoff value greatly changes the ultimate image quality, as requiring a high $(>0.5 \mathrm{MeV})$ cutoff results in few cones with low uncertainty per cone, while a low cutoff results in many cones with high uncertainty per cone.

The pulses remaining after the application of the energy and timing filters are fed into a 'for' loop to identify neutron double scatter candidates. A voltage pulse resulting from a double neutron scatter in a small, ideal scintillator detector should resemble a large voltage pulse followed by a second pulse several nanoseconds later. The second pulse rise will begin before the first pulse has a full time to decay to baseline. In most scintillator detectors, the second pulse will not be distinguishable from the first, both because of the long light scintillation time and/or because the sampling rate is too low to digitize the second scatter features. Both of these scenarios result in double scatters that resemble single pulses. The simplified NSC system can distinguish pulses that occur several nanoseconds apart through the use of a fast timing scintillator material and a sub-nanosecond digitizer. The first step in searching for these double scatter events is establishing a timing window in which it is possible for secondary scatters to appear. The reported pulse width for the EJ-230 material is $1.3 \mathrm{~ns}$, and it is known that any photodetectors will add some amount of timing blur to the that width. Additionally, even if pulses very close together in time can be resolved, high uncertainty due to their low times-of-flight will result in probability cones with high errors in kinematic properties. Due to these facts, it was assumed that the window for possible second peaks should begin at $2 \mathrm{~ns}$ after the first peak in each voltage pulse. The distribution in time difference between scatter positions 1 and 2 is known from Monte Carlo simulation. This distribution provides guidance on the end of the time window. Virtually no double scatters with a time separation of greater than $12 \mathrm{~ns}$ occur in a detector of this size. This means that a window for a second peak beyond that upper value would likely be incorporating secondary particle coincidences, which results in erroneous cones. Based on these material and empirical considerations, the timing window for the system was set at 2 to $12 \mathrm{~ns}$ after the first scatter peak. Figure 7 shows a candidate double pulse as seen by one PMT with the timing window demonstrated.

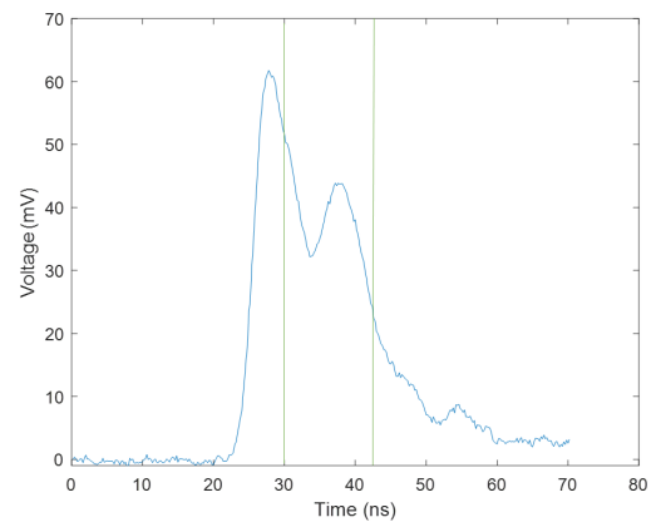

Fig. 7. Timing window for neutron double scatter candidates. The peak of the secondary scatter feature must appear between 2 and $12 \mathrm{~ns}$ after the primary peak. 
With the timing window established, the next parameter to determine is the empirical value related to the secondary feature necessary for the event to be considered a true second neutron scatter. We will call this the second scatter criterion. It is relevant to determine whether the second scatter criterion should be applied to each pulse individually, or only to a select few from each sestet of coincident pulses. A number of definitions for the second scatter criterion were applied in a trial-and-error manner to data sets, as there is little guidance in this area in the literature.

For the testing of each second scatter criterion definition, the NSC system measured a ${ }^{252} \mathrm{Cf}$ source at a known location for six hours. Each second scatter criterion definition was applied to the data set to yield an array of double scatter candidates. Section III of the script was then run on each resulting double scatter candidate array to generate neutron images. The neutron images resulting from the differing definitions were analyzed for convergence to the neutron source position over a range of energy deposition cutoff values and second scatter criterion values according to their definitions. The results from these trials are summarized in Table I. It was found that the two peak prominence ratio method yielded images that converged to the correct source position most often. A visualization of this method is shown in Figure 8. TABLE I

DESCRIPTION AND PERFROMANCE OF SECOND SCATTER CRITERIA

\begin{tabular}{|c|c|c|}
\hline Second scatter criterion & Descrintion & Performance \\
\hline $\begin{array}{l}\text { Second peak absolute } \\
\text { prominence }\end{array}$ & $\begin{array}{l}\text { Prominence of highest peak in the time window } \\
\text { must pass an absolute cutoff value. }\end{array}$ & Fair \\
\hline Positive gradient & $\begin{array}{l}\text { Derivative of voltage values in the time window is } \\
\text { found and must pass cutoff indicating that the } \\
\text { voltage in at least part of the window has a positive } \\
\text { slope. }\end{array}$ & Poor \\
\hline Two peak prominence ratio & $\begin{array}{l}\text { Prominence of pulse maximum and peak maximum } \\
\text { in the time window are found. Ratio of the peak } \\
\text { prominence of the two must pass a cutoff value } \\
\text { indicating the second peak has a prominence that } \\
\text { is greater than or equal to a certain fraction of the } \\
\text { prominence of the first. }\end{array}$ & Good \\
\hline Forced Gaussian fit & $\begin{array}{l}\text { Each pulse with a peak in the time window is fit with } \\
\text { a Gaussian centered at that peak. The goodness- } \\
\text { of-fit value for the fit must pass a cutoff to be } \\
\text { considered a true second scatter. }\end{array}$ & Poor \\
\hline Selection by visual inspection & $\begin{array}{l}\text { Pulses with a peak in the time window are saved, } \\
\text { and then each remaining is visually inspected. } \\
\text { Pulses that visually look like noise or coincidence } \\
\text { are eliminated. }\end{array}$ & $\begin{array}{l}\text { Fair, but time } \\
\text { consuming }\end{array}$ \\
\hline
\end{tabular}

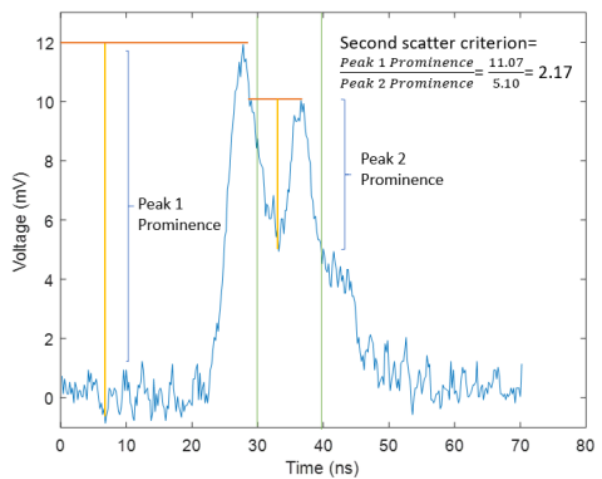

Fig. 8. Demonstration of second scatter criterion using the two peak prominence ratio method. In generating the neutron images to evaluate this method, the second scatter criterion for this method was ranged over values between 1.5 and 10 .

This method for second scatter identification was implemented in the code so that only one pulse each sestet of coincident pulses needed to fulfill the second scatter criterion for the entire sestet to be accepted as a double scatter candidate. It was found that when generating test images using this method, requiring all six or some combination of pulses to meet the second scatter criterion resulted in double scatter candidate data sets with much fewer pulses than the simulated double scatter efficiency suggested, indicating a high rate of false negatives. Only requiring any one of the pulses in each sestet to meet the criterion greatly lowered this false negative rate.

Determining scatter position 1 begins by finding the voltage maximum in the timing window where scatter 1 is expected to be for each pulse. This window is defined as plus or minus $2 \mathrm{~ns}$ from the average pulse maximum timing bin location for that digitizer channel. The maximum voltage values are then compared with the values generated by the same event in the opposite PMT channel by taking the log ratio of the pulse heights and then plugging those value into the position calibration curves presented in Figure 3. Doing this for each pair of PMTs yields scattering position of first scatter events for each double scatter candidate, labeled $\mathrm{x}_{1}, \mathrm{y}_{1}$, and $\mathrm{z}_{1}$ in the script.

Second scattering positions are found in a similar manner, though they require the extra step of subtracting the elevated baseline caused by the first scatter just nanoseconds before. For each double scatter candidate pulse, a Gaussian fit is performed on the second peak to estimate the pulse height that would be caused by that scattering event had the first scatter not occurred. The height from the signal baseline to the maximum of the Gaussian is then used as the second peak height, which is subsequently applied to the log ratio and then the calibration curve. A visual demonstration of this process for double scatter candidate pulses from opposite PMTs is shown in Figure 9.
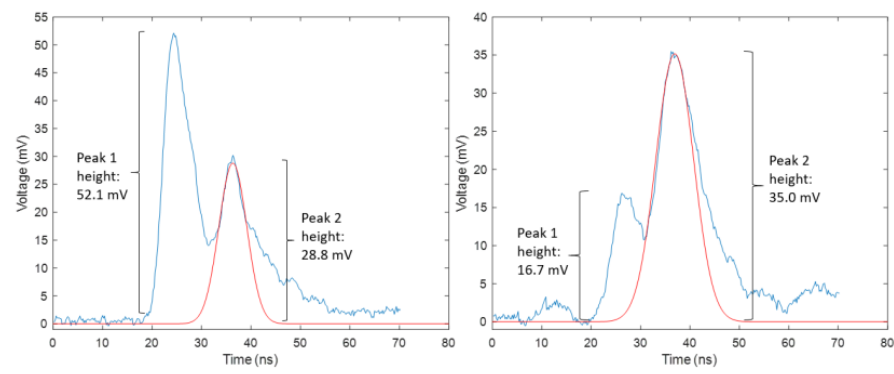

Fig. 9. Peak height determination for neutron double scatter candidate pulses. These pulses were produced by the pair of PMTs mounted to opposite ends of the camera along the $\mathrm{x}$-axis of measurement. Taking the $\log$ ratios of the peak 1 and peak 2 heights yields scatter positions of +1.85 inches and -0.20 inches from the detector centerline in the $\mathrm{x}$ direction for scatters 1 and 2, respectively.

The energy deposited by the first neutron scatter, $E_{f}$, is determined running the peak 1 height values for a sestet through the energy calibration curves presented in Figure 4, averaging over each of the six-correlated pulses for the event, and then applying the electron-equivalent energy deposition values to the neutron light curve presented in Figure 5. The error for each $E_{f}$ value is found by assuming that the production of light in the scintillator is a Poisson process, and thus the number of photoelectrons generated and the voltage pulse heights have an error proportional to the square root of their respective values. The errors of the pulse height are then propagated through the energy calibration curve and the light curve to yield an array of $\sigma_{\text {Ef values. }}$

The proton recoil energies and the first and second neutron scatter positions can be used with Equations 2, 3, and 4 and 
standard error propagation rules to find all the values necessary for probability cone back-projection.

\section{RESULTS}

\section{A. Point Source Measurements}

A small $45 \mu \mathrm{Ci}{ }^{252} \mathrm{Cf}$ spontaneous fission source was measured at a variety of angles relative to the camera to test the system's localization ability. Measurements of 5 million total counts at two different spatial positions resulted in the raw neutron images shown in Figures 10 and 11. The high amount of noise resulting from erroneously oriented cones leads the raw images to converge to incorrect positions or feature multiple probability hotspots, reducing the image usefulness. Erroneous cones can be eliminated by down-selecting the cones used to produce the raw images according to an initial source direction guess vector. A guess vector can predict a likely direction of a neutron source by comparing the skew in the distribution of opposite PMT light ratios for all events detected in the scintillator volume during the measurement time. The skewness of log light ratios can be compared to values in a lookup table based on reference measurements of the camera with the same calibration settings. Once a guess vector is made, a region around it is formed on the spherical projection surrounding the camera system. All back-projected probability cones that do not intersect the guess vector region are then removed from the neutron image, resulting in cleaner and better resolved images free of incorrectly oriented cone. Figure 12 visually demonstrates the process of image improvement using guess vector down-selection. Figures 13 and 14 show "improved" neutron images that use the guess vector down-selection technique to create symmetric and well-resolved images.

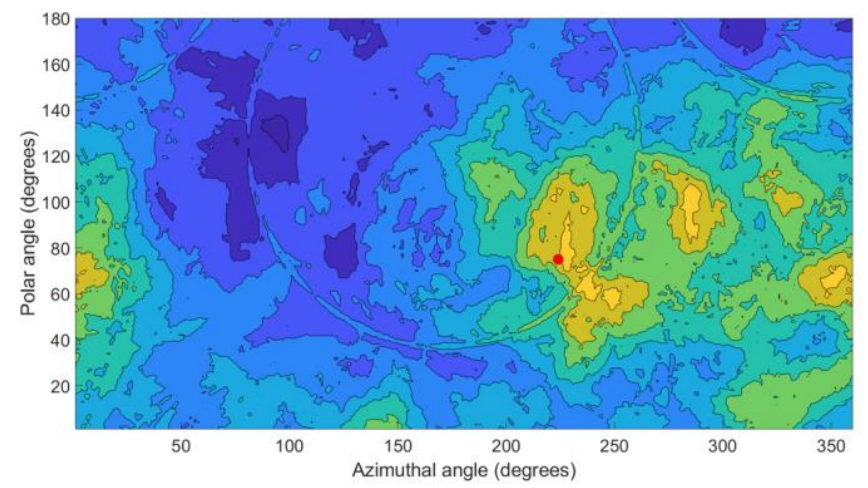

Fig. 10. Raw neutron image of point source measured at $(224,75)$. The image was generated with a first scatter energy deposition cutoff of $0.2 \mathrm{MeV}$ and a peak prominence ratio cutoff of 4.154 cones were used to generate the image.

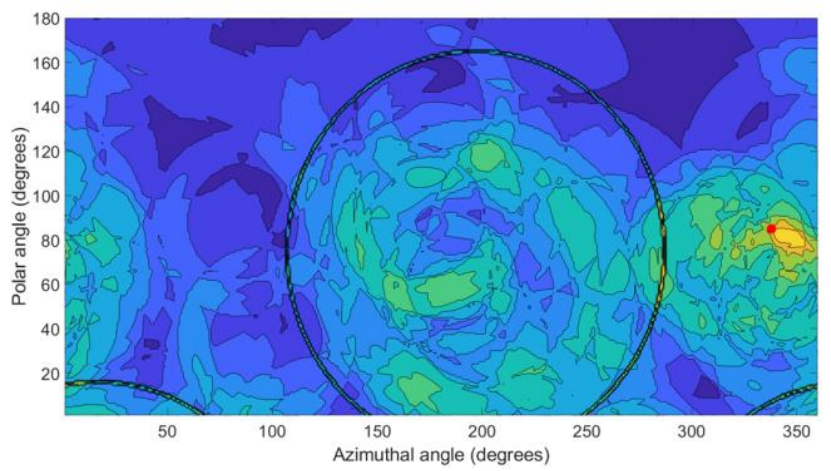

Fig. 11. Raw neutron image of point source 2 measured at source position 2 . The image was generated with a first scatter energy deposition cutoff of $0 \mathrm{MeV}$ and a peak prominence ratio cutoff of 2.42 cones were used to generate the image.
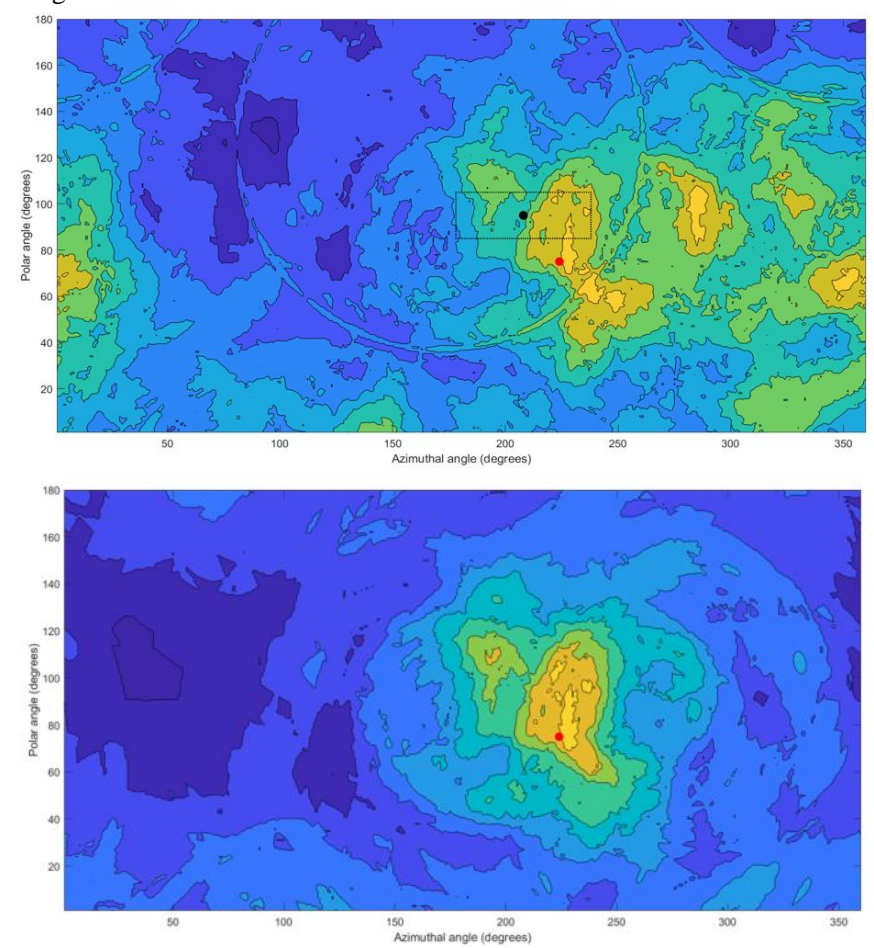

Fig. 12. Demonstration of cone down-selection using source direction guess vector region. All cones that do not overlap the region oullined in black in the first image are removed to form the improved image.

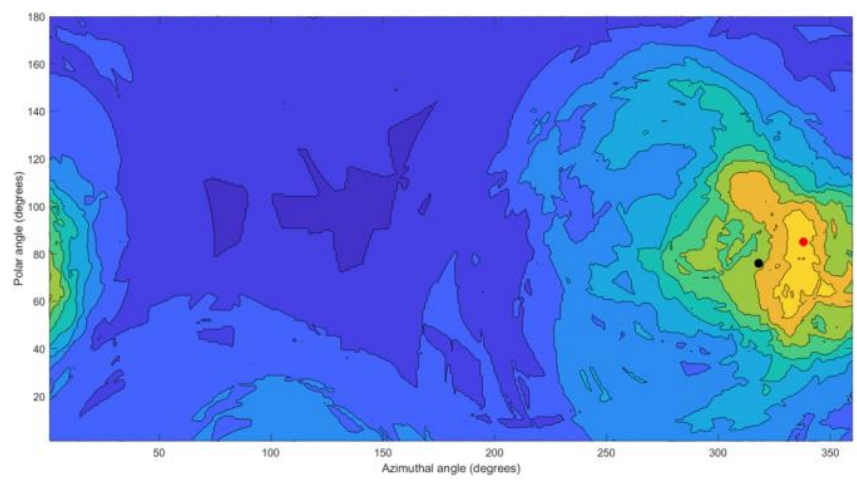

Fig. 13. Improved neutron image of a point source located at $(338,85)$. The initial guess vector points towards $(318,76)$. The image was made with an initial energy deposition cutoff of $0.5 \mathrm{MeV}$, a second scatter criterion prominence ratio of 6.5 , and 48 cones. The guess vector region was set to $60^{\circ}$ azimuthal width and $20^{\circ}$ polar height.

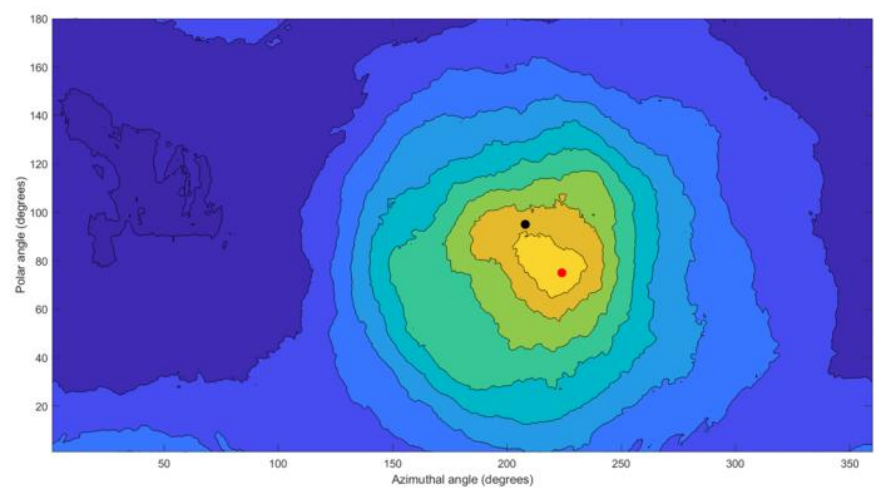

Fig. 14. Improved neutron image of a point source located at $(224,75)$. The 
initial guess vector points towards $(208,95)$. The image was made with an initial energy deposition cutoff of $0 \mathrm{MeV}$, a second scatter criterion prominence ratio of 4 , and 654 cones. The guess vector region was set to $60^{\circ}$ azimuthal width and $20^{\circ}$ polar height.

\section{B. Camera Performance}

The imaging resolution of a camera-type radiation detector can be assessed through source position cross-cutting. The camera system does not have a single imaging resolution but rather a range of possible resolutions that vary with energy deposition cutoffs, second scatter criterion, guess vector downselection use, and guess vector down-selection region size. To assess this range of resolutions, the data from the point source measured at $(18.3,224,75)$ was iteratively run through the neutron image creation portion of the script while varying both the energy deposition cutoff and the second scatter criterion. A series of images corresponding to each cutoff value was saved for both the raw images and improved images (those that used guess vector down-selection). The resolution of each image was then determined by crosscutting each of the images in the polar and azimuthal directions at the known source location. In many cases, the peak of these crosscuts did not correspond to the true source direction. In these cases, the azimuthal and polar error between the true source position and the images' maximum probability pixel are recorded. Table II lists the resolution values, localization errors, and number of probability cones for a range of improved neutron images. In cases where multiple probability hotspots appeared along a crosscut, the peak corresponding to the hotspot nearest to the true source position was used for resolution and error determination. Images were determined to have "no convergence" if a hotspot of at least $80 \%$ of the probability of the hottest projection bin was not within 60 degrees of the true source position.

\section{TABLE II}

\begin{tabular}{|c|c|c|c|c|}
\hline \multicolumn{5}{|c|}{ st S } \\
\hline $\begin{array}{l}\text { Peak } \\
\text { prominence } \\
\text { ratio cutoff }\end{array}$ & 0 & 0.2 & 0.4 & 0.6 \\
\hline 5 & $(44,1,38,9,413)$ & $(49,16,41,4,183)$ & $(54,33,41,17,55)$ & $(30,30,20,13,16)$ \\
\hline 4.5 & $(42,13,40,5,289)$ & $(39,20,39,11,116)$ & $(56,18,29,14,33)$ & $(38,9,26,13,12)$ \\
\hline 4 & $(31,20,43,15,176)$ & $(43,16,29,3,87)$ & $(44,23,29,17,25)$ & No convergence \\
\hline 3.5 & $(31,1,31,3,104)$ & $(30,22,24,3,42)$ & No convergence & No convergence \\
\hline 3 & $(43,23,34,10,67)$ & $(40,23,31,16,30)$ & $(31,8,25,7,9)$ & No convergence \\
\hline 2.5 & $(54,22,37,6,35)$ & $(37,19,23,17,17)$ & No convergence & No convergence \\
\hline 2 & $(30,3,45,2,23)$ & No convergence & No convergence & No convergence \\
\hline 1.5 & $(20,3,23,10,13)$ & No convergence & No convergence & No convergence \\
\hline
\end{tabular}

Resolutions, convergence position errors, and number of cones for raw neutron images generated at a range of energy deposition and peak prominence ratio cutoffs. The data in each cell is reported in the form: (azimuthal resolution, azimuthal error, polar resolution, polar error, number of cones). The resolutions and errors are reported in degrees.

Like the resolution determination, the camera system will not have a single double scatter efficiency but rather a range of achievable efficiencies determined by the cutoff values used in determination of which pulse sestets are used in cone generation.

To test the camera system's useful cone efficiency, a measurement of 12 million counts generated from the two ${ }^{252} \mathrm{Cf}$ point sources together was run iteratively over a range of cutoffs. The sources were position together and shielded with three lead bricks to simulate a "pure" neutron source free from non-background gamma rays. The datafile was iteratively run through section 2 of the double pulse identification algorithm, with either the peak prominence ratio cutoff or the first scatter energy deposition cutoff changed at each step. At each iteration, the useful cone efficiency for those settings was determined by divided the double scatter candidate identified by the total counts in the datafile. Figure 15 shows a series of useful cone efficiency curves at different cutoff values. As the first scatter energy depostion cutoff value is increased, the useful cone efficency decreases in a log linear fashion across the range of peak prominence ratio cutoffs. These curves can be used to guide the selection of cutoff values for a desired count rate or measurment time as needed.

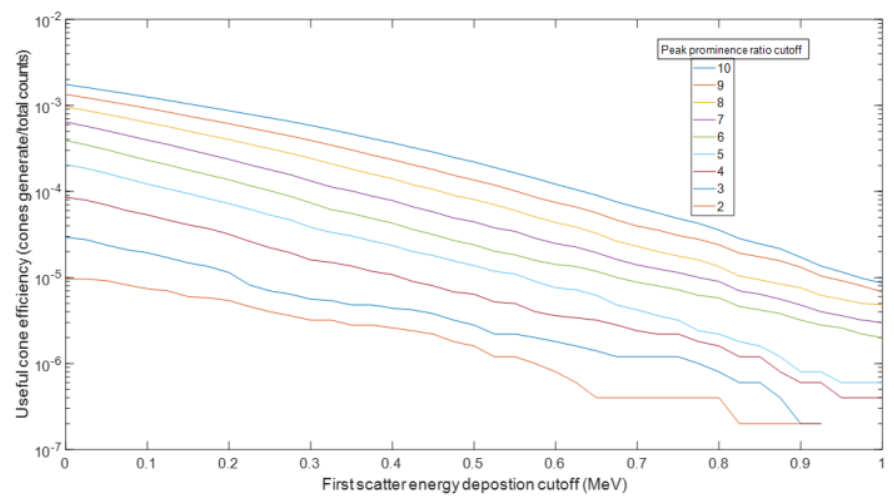

Fig. 15. Useful cone efficiency curves for the camera system prototype.

\section{CONCLUSIONS}

The prototype simplified neutron scatter camera described in the proceeding sections has shown promising results for the localization of neutron sources given measurement times on the order of several hours. Based on the data shown in the proceeding sections and observations made during data analysis, the following is a non-exhaustive list of the successes of the simplified neutron scatter camera prototype:

- The combination of a short light pulse scintillator material with sub-nanosecond digitizer timing allows the system to resolve scattering events just nanoseconds apart across six PMTs simultaneously. Ratios of the amount of fluorescence light arriving at opposite PMTs coupled to a single volume can be used to reconstruct neutron scatter positions within the scintillator volume with errors on the order of millimeters. No use of pixelated photomultipliers or optical segmentation is necessary for position reconstruction.

Simulation scaling studies show that scatter position reconstruction using the light ratios method functions at variety of scintillator cube volumes, making it possible to construct detectors with this design with a range of sizes.

The algorithm developed to identify neutron double scatter candidates based on analysis of pulse characteristics was successful. Several different criteria for second scatter identification showed some successes, with a comparison of first and second peak prominence empirically the most successful.

- $\quad$ Double scatter candidate pulses successfully localize neutron point and volume sources over a range of spatial positions, and at a variety of energy and double scatter criterion cutoffs. 
- Guess vector probability cone down-selection improves neutron images by removing erroneous cones, leading to images with better resolution and fewer incorrect localization positions.

- Guess vector method can give users of the simplified NSC a general idea of an unknown source direction before any cone back-projection is performed.

- The entire system is significantly less expensive per unit that other designs for neutron scatter cameras due to the use of fewer photomultipliers and a single unsegmented scintillator volume.

With further refinement of the double scatter identification algorithm, optimizing of the detector volume and photomultiplier size, explorations of different scintillator materials, reflectors, and photomultipliers, and the honing of the imaging down-selection method, a detector using the Simplified Neutron Scatter Camera design could become a valuable tool for international nuclear safeguards.

\section{REFERENCES}

[1] International Atomic Energy Agency. "Basics of IAEA Safeguards," [Online] Available: https://www.iaea.org/topics/basics-of-iaeasafeguards. Accessed on" June 30, 2021.

[2] J.E. Doyle. "Introduction: Nuclear Security and Global Challenges," in Nuclear Safeguards, Security, and Nonproliferation, $1^{\text {st }}$ ed. Burlington, MA, USA: Butterworth-Heinmann, 2008.

[3] R.C. Runkle, A. Bernstein, and P.E. Vanier, "Securing Special Nuclear Material: Recent Advances in Neutron Detection and Their Role in Nonproliferation," Journal of Applied Physics, vol. 108, no. 11, 2010, 10.1063/1.3503495.

[4] D. Xu, Z. He, C.E. Lehner, F. Zhang. (2004, August). 4-pi Compton Imaging with Single 3D Position Sensitive CdZnTe Detector. Presented at SPIE 5540, Hard X-Ray and Gamma-Ray Detector Physics VI. [Online]. Available: doi.org/10.1117/12.563905. Accessed on: June 30, 2021.

[5] N. Mascarenhas, J. S. Brennan, and K. Krenz. (2006, October). Development of a Neutron Scatter Camera for Fission Neutrons. Presented at: 2006 IEEE Nuclear Science Symposium. [Online]. Available: https://www.semanticscholar.org/paper/Development-of-aNeutron-Scatter-Camera-for-Fission-MascarenhasBrennan/a3544f4fbce0f4c048d11c2577ad2ab3d0ddb0e7. Accessed on: June 30, 2021.

[6] J. E. M. Goldsmith, M.D. Gerling, J.S. Brennan, “A compact neutron scatter camera for field deployment," Review of Scientific Instruments, Vol. 87, August 2016, DOI. 10.1063/1.4961111.

[7] K. Weinfurther, J. Mattingly, E. Brubaker, J. Steele, "Model-Based Design Evaluation of a Compact, High-Efficiency Neutron Scatter Camera," Nuclear Instruments and Methods in Physics Research Section A, Vol. 883, pp. 115-135. March 2018. DOI. 10.1016/j.nima.2017.11.025.

[8] J.J. Manfredi, B.L. Goldblum, T.A. Laplace, G. Gabella, J. Gordon, A. O’Brien, S. Chowdhury, J.A. Brown, and E. Brubaker, "Proton Light Yield of Fast Plastic Scintillators for Neutron Imaging," IEEE Transactions in Nuclear Science, vol. 67, no. 2, pp. 434-442, December 2019. DOI. 10.1109/TNS.2019.2959979. 